\title{
PROPOSITIONAL LOGIC WORD PROBLEMS AND MS EXCEL AT PRIMARY AND SECONDARY EDUCATION
}

\author{
Jitka Laitochová, David Nocar, \& Karel Pastor \\ Department of Mathematics, Faculty of Education, Palacký University (Czech Republic)
}

\begin{abstract}
It seems that propositional logic word problems are interesting for pupils aged 9 to 16 years. Younger pupils usually solve the propositional logic word problems by means of the systematic analysis of possible cases. In this way they significantly develop their combinatorial skills.

Nevertheless, we can solve the propositional logic word problems almost automatically using logical connectives and truth tables. Moreover, there are software applications for solving problems of mathematical logic. For example, MS Excel can help to work with the truth table.

We carried out research with the aid of a questionnaire to find out the preparedness of prospective elementary teachers to use MS Excel for solving propositional logic word problems.

In our paper we show some examples of propositional logic word problems for pupils of different ages. In our opinion, using MS Excel for solving propositional logic word problems can make such word problems even more attractive.
\end{abstract}

Keywords: Propositional logic, word problems, MS Excel.

\section{Introduction}

Example 1. Kattie, Lucy, and Emily are quarrelling triplet. If Kattie goes on the trip, then Lucy will not go. If Emily goes on the trip, then Lucy will not go. Kattie or Emily will go on the trip. Lucy or Emily will go on the trip. Can a couple of girls go on that trip?

The previous problem can be solved even by younger pupils aged 9 or 10 years by means of systematic analysis of three possible cases:

1. Kattie and Lucy will go together on the trip.

2. Kattie and Emily will go together on the trip.

3. Lucy and Emily will go together on the trip.

It is very simple to verify given conditions that only Kattie and Emily can go together on the trip.

Nevertheless, we can solve the previous propositional logic word problem using logical connectives and truth table.

In our paper we recall the most common logical connectives (Section 2) and explain how to use MS Excel to create a truth table for solving a propositional logic word problem.

\section{Logical connectives}

By means of ("Logical connective", Wikipedia) we recall the most used logical connectives. Moreover, we use ("Using logical functions", 2018) to show how MS Excel can help in creating truth tables of logical connectives.

\subsection{Negation}

Negation is an operation on one logical value, typically the value of a proposition that produces a value of true when its operand is false and a value of false when its operand is true. So, if a statement $P$ is true, then $\neg P$ (pronounced "not $P$ ") would therefore be false; and conversely, if $P$ is false, then $\neg P$ would be true. 
Table 1. Truth table of negation.

\begin{tabular}{|c|c|}
\hline$P$ & $\neg P$ \\
\hline 0 & 1 \\
\hline 1 & 0 \\
\hline
\end{tabular}
function:

In the previous truth table 0 means false and 1 means true. In MS Excel, we can use the IF

Table 2. Truth table of negation generated by MS Excel.

\begin{tabular}{|c|c|c|}
\cline { 2 - 3 } \multicolumn{1}{c|}{} & $\mathbf{A}$ & $\mathbf{B}$ \\
\hline $\mathbf{1}$ & $P$ & $\neg P$ \\
\hline $\mathbf{2}$ & 0 & $=\mathrm{IF}(\mathrm{A} 2=0 ; 1 ; 0)$ \\
\hline $\mathbf{3}$ & 1 & $=\mathrm{IF}(\mathrm{A} 3=0 ; 1 ; 0)$ \\
\hline
\end{tabular}

\subsection{Conjuction}

Logical conjunction is an operation on two logical values, typically the values of two propositions, that produces a value of true if and only if both of its operands are true. Conjuction of two statements $P, R \quad$ is denoted by $P \wedge Q$ (pronounced " $P$ and $R$ ").

Table 3. Truth table of conjuction.

\begin{tabular}{|c|c|c|}
\hline$P$ & $R$ & $P \wedge R$ \\
\hline 0 & 0 & 0 \\
\hline 0 & 1 & 0 \\
\hline 1 & 0 & 0 \\
\hline 1 & 1 & 1 \\
\hline
\end{tabular}

In MS Excel, we can use the IF function together with the AND function:

Table 4. Truth table of conjuction generated by MS Excel.

\begin{tabular}{|c|c|c|l|}
\cline { 2 - 4 } \multicolumn{1}{c|}{} & $\mathbf{A}$ & $\mathbf{B}$ & \multicolumn{1}{c|}{ C } \\
\hline $\mathbf{1}$ & $P$ & $R$ & $P \wedge R$ \\
\hline $\mathbf{2}$ & 0 & 0 & =IF(AND(A2;B2)=TRUE;1;0) \\
\hline $\mathbf{3}$ & 0 & 1 & =IF(AND(A3;B3)=TRUE;1;0) \\
\hline $\mathbf{4}$ & 1 & 0 & =IF(AND(A4;B4)=TRUE;1;0) \\
\hline $\mathbf{5}$ & 1 & 1 & =IF(AND(A5;B5)=TRUE;1;0) \\
\hline
\end{tabular}

\subsection{Disjunction}

Logical disjunction is an operation on two logical values, typically the values of two propositions, that has a value of false if and only if both of its operands are false. Disjunction of two statements $P, R$ is denoted by $P \vee R$ (pronounced " $P \vee R$ ").

Table 5. Truth table of disjunction.

\begin{tabular}{|c|c|c|}
\hline$P$ & $R$ & $P \vee R$ \\
\hline 0 & 0 & 0 \\
\hline 0 & 1 & 1 \\
\hline 1 & 0 & 1 \\
\hline 1 & 1 & 1 \\
\hline
\end{tabular}

In MS Excel, we can use the IF function together with the OR function: 
Table 6. Truth table of disjuction generated by MS Excel.

\begin{tabular}{|c|c|c|c|}
\cline { 2 - 4 } \multicolumn{1}{c|}{} & A & B & C \\
\hline $\mathbf{1}$ & $P$ & $R$ & $P \wedge R$ \\
\hline $\mathbf{2}$ & 0 & 0 & =IF(OR(A2;B2)=TRUE;1;0) \\
\hline $\mathbf{3}$ & 0 & 1 & $=\mathrm{IF}(\mathrm{OR}(\mathrm{A} 3 ; \mathrm{B} 3)=\mathrm{TRUE} ; 1 ; 0)$ \\
\hline $\mathbf{4}$ & 1 & 0 & $=\mathrm{IF}(\mathrm{OR}(\mathrm{A} 4 ; \mathrm{B} 4)=\mathrm{TRUE} ; 1 ; 0)$ \\
\hline $\mathbf{5}$ & 1 & 1 & $=\mathrm{IF}(\mathrm{OR}(\mathrm{A} 5 ; \mathrm{B} 5)=\mathrm{TRUE} ; 1 ; 0)$ \\
\hline
\end{tabular}

\subsection{Implication}

Implication is used to form statements of the form $P \Rightarrow R$ which is read "if $P$ then $R$ ". $P$ is termed the antecedent of the implication and $R$ is termed the consequent of the implication. The implication $P \Rightarrow R$ is false if and only if $P$ is true and $R$ is false.

Table 7. Truth table of implication.

\begin{tabular}{|c|c|c|}
\hline$P$ & $R$ & $P \Rightarrow R$ \\
\hline 0 & 0 & 1 \\
\hline 0 & 1 & 1 \\
\hline 1 & 0 & 0 \\
\hline 1 & 1 & 1 \\
\hline
\end{tabular}

In MS Excel, we can use the IF function by the following way:

Table 8. Truth table of implication generated by MS Excel.

\begin{tabular}{|c|c|c|c|}
\cline { 2 - 4 } \multicolumn{1}{c|}{} & A & B & C \\
\hline $\mathbf{1}$ & $P$ & $R$ & $P \Rightarrow R$ \\
\hline $\mathbf{2}$ & 0 & 0 & $=\mathrm{IF}(\mathrm{IF}(\mathrm{F} 1 ; \mathrm{G} 1=1 ;$ TRUE)=TRUE;1;0) \\
\hline $\mathbf{3}$ & 0 & 1 & $=\mathrm{IF}(\mathrm{IF}(\mathrm{F} 2 ; \mathrm{G} 2=1 ; \mathrm{TRUE})=\mathrm{TRUE} ; 1 ; 0)$ \\
\hline $\mathbf{4}$ & 1 & 0 & $=\mathrm{IF}(\mathrm{IF}(\mathrm{F} 3 ; \mathrm{G} 3=1 ;$ TRUE)=TRUE;1;0) \\
\hline $\mathbf{5}$ & 1 & 1 & $=\mathrm{IF}(\mathrm{IF}(\mathrm{F} 4 ; \mathrm{G} 4=1 ;$ TRUE)=TRUE;1;0) \\
\hline
\end{tabular}

\subsection{Logical equality}

Logical equality is an operation on two logical values, typically the values of two propositions, that produces a value of true if and only if both operands are false or both operands are true. Logical equality of two statements $P, R$ is denoted by $P \Leftrightarrow R$ (pronounced " $P$ iff $R$ ").

Table 9. Truth table of logical equality.

\begin{tabular}{|c|c|c|}
\hline$P$ & $R$ & $P \Leftrightarrow R$ \\
\hline 0 & 0 & 1 \\
\hline 0 & 1 & 0 \\
\hline 1 & 0 & 0 \\
\hline 1 & 1 & 1 \\
\hline
\end{tabular}

Now, the IF function in MS Excel can be used very simply:

Table 10. Truth table of implication generated by MS Excel.

\begin{tabular}{|r|c|c|c|}
\cline { 2 - 4 } \multicolumn{1}{c|}{} & A & B & \multicolumn{1}{c|}{ C } \\
\hline $\mathbf{1}$ & $P$ & $R$ & \multicolumn{1}{c|}{$P \Leftrightarrow R$} \\
\hline $\mathbf{2}$ & 0 & 0 & $=\mathrm{IF}(\mathrm{F} 1=\mathrm{G} 1 ; 1 ; 0)$ \\
\hline $\mathbf{3}$ & 0 & 1 & $=\mathrm{IF}(\mathrm{F} 2=\mathrm{G} 2 ; 1 ; 0)$ \\
\hline $\mathbf{4}$ & 1 & 0 & $=\mathrm{IF}(\mathrm{F} 3=\mathrm{G} 3 ; 1 ; 0)$ \\
\hline $\mathbf{5}$ & 1 & 1 & $=\mathrm{IF}(\mathrm{F} 4=\mathrm{G} 4 ; 1 ; 0)$ \\
\hline
\end{tabular}




\section{Examples}

Now, using MS Excel eventually, we can create a truth table for Example 1. We denote by K the proposition "Kattie will go on the trip", by E the proposition "Emily will go on the trip", and finally we denote by L the proposition "Lucy will go on the trip".

Table 11. Truth table of Example 1.

\begin{tabular}{|c|c|c|c|c|c|c|}
\hline $\mathbf{K}$ & $\mathbf{E}$ & $\mathbf{L}$ & $\mathbf{K} \Rightarrow \neg \mathbf{L}$ & $\mathbf{E} \Rightarrow \neg \mathbf{L}$ & $\mathbf{K} \vee \mathbf{L}$ & $\mathbf{L} \vee \mathbf{E}$ \\
\hline 0 & 0 & 0 & 1 & 1 & 0 & 0 \\
\hline 0 & 0 & 1 & 1 & 1 & 1 & 1 \\
\hline 0 & 1 & 0 & 1 & 1 & 0 & 1 \\
\hline 1 & 0 & 0 & 1 & 1 & 1 & 0 \\
\hline 0 & 1 & 1 & 1 & 0 & 1 & 1 \\
\hline 1 & 0 & 1 & 0 & 1 & 1 & 1 \\
\hline 1 & 1 & 0 & 1 & 1 & 1 & 1 \\
\hline 1 & 1 & 1 & 0 & 0 & 1 & 1 \\
\hline
\end{tabular}

Of course, the use of MS Excel is more advantageous in the case of a larger number of examined variables. The following example is intended rather for older pupils aged from 14 to 16 years.

Example 2. A handball team coach is thinking about a line-up for a match. He is taking the following conditions into account:

1. If player $\mathrm{K}$ plays, then player $\mathrm{L}$ will play.

2. If player $\mathrm{O}$ plays, then players $\mathrm{K}$ and $\mathrm{N}$ will play.

3. Players $\mathrm{L}$ and $\mathrm{M}$ will not play together.

4. Player $\mathrm{M}$ will play if and only if player $\mathrm{N}$ will play.

5. Player $\mathrm{N}$ or player $\mathrm{O}$ will play.

Who of the players $\mathrm{K}, \mathrm{L}, \mathrm{M}, \mathrm{N}, \mathrm{O}$ will play?

We can use MS Excel to create a truth table. With respect to its extant (it has $1+32$ lines), we show only its part:

Table 12. Truth table of Example 2.

\begin{tabular}{|c|c|c|c|c|c|c|c|c|c|}
\hline $\mathbf{K}$ & $\mathbf{L}$ & $\mathbf{M}$ & $\mathbf{N}$ & $\mathbf{O}$ & $\mathbf{K} \Rightarrow \mathbf{L}$ & $\mathbf{O} \Rightarrow(\mathbf{K} \wedge \mathbf{N})$ & $\neg(\mathbf{L} \wedge \mathbf{M})$ & $\mathbf{M} \Leftrightarrow \mathbf{N}$ & $\mathbf{N} \vee \mathbf{O}$ \\
\hline 0 & 0 & 0 & 0 & 0 & 1 & 1 & 1 & 1 & 0 \\
\hline 0 & 0 & 0 & 0 & 1 & 1 & 0 & 1 & 1 & 1 \\
\hline$\cdot$ & $\cdot$ & $\cdot$ & $\cdot$ & $\cdot$ & $\cdot$ & $\cdot$ & $\cdot$ & $\cdot$ & $\cdot$ \\
\hline$\cdot$ & $\cdot$ & $\cdot$ & $\cdot$ & $\cdot$ & $\cdot$ & $\cdot$ & $\cdot$ & $\cdot$ & $\cdot$ \\
\hline 1 & 0 & 0 & 0 & 1 & 0 & 0 & 1 & 1 & 1 \\
\hline 0 & 0 & 1 & 1 & 0 & 1 & 1 & 1 & 1 & 1 \\
\hline 0 & 1 & 0 & 1 & 0 & 1 & 1 & 1 & 0 & 1 \\
\hline$\cdot$ & $\cdot$ & $\cdot$ & $\cdot$ & $\cdot$ & $\cdot$ & $\cdot$ & $\cdot$ & $\cdot$ & $\cdot$ \\
\hline$\cdot$ & $\cdot$ & $\cdot$ & $\cdot$ & $\cdot$ & $\cdot$ & $\cdot$ & $\cdot$ & $\cdot$ & $\cdot$ \\
\hline 1 & 1 & 1 & 1 & 0 & 1 & 1 & 0 & 1 & 1 \\
\hline 1 & 1 & 1 & 1 & 1 & 1 & 1 & 0 & 1 & 1 \\
\hline
\end{tabular}

\section{Research}

A total of 67 prospective elementary teachers studying at the Faculty of Education in Olomouc, Czech Republic, answered the following simple question (after the propositional logic lessons):

Had an idea of using MS Excel come to you during solving propositional logic word problems?

On this question only 8 students answered yes and 59 students answered no. As much attention is paid to MS Excel at primary and secondary schools, the previous result is not very positive. 


\section{Acknowledgements}

The research is supported by Palacký University in Olomouc, Czech Republic (IGA_PdF_2019_001).

\section{References}

Cheusheva, S. (23-08-2018). Using logical functions in Excel: AND, OR, XOR and NOT. Retrieved 13-05-2019, from https://www.ablebits.com/office-addins-blog/2014/12/17/excel-and-or-xor-notfunctions/

(n.d.). Logical connective (Wikipedia). Retrieved 04-05-2019, from https://en.wikipedia.org/wiki/Logical_connective 